\title{
A Post-processing Technique to Suppress Fluid Signal and Increase Contrast in Multispectral MR Exams of MS Patients
}

\author{
J.R. Mitchell ${ }^{1,2,3}$, P. Gareau ${ }^{1}$, S. Karlik ${ }^{1,2,3}$, B. Rutt ${ }^{1,2,3}$ \\ Imaging Research Laboratories, John P. Robarts Research Institute ${ }^{1}$; Department of Diagnostic \\ Radiology and Nuclear Medicine, University of Western Ontario ${ }^{2}$, and, The London Health \\ Sciences Center ${ }^{3}$, London Ontario, Canada N6A 5A5
}

\begin{abstract}
We present a new method to extract data from multispectral MR exams of patients with Multiple Sclerosis. Our technique produces images of "spectral phase" relative to cerebro-spinal fluid (CSF-SP images). It provides a convenient way of reducing multispectral MR exams to a single, intuitive image with contrast characteristics similar to anatomical photographs. Our new images provide better tissue contrast than that found in any of the MR images. Contrast between CSF and white matter (WM) was increased from a maximum of 19.5 in the T1w MR image to 56 in the CSF-SP image (+187\%). Contrast between CSF and gray matter $(\mathrm{GM})$ increased from a maximum of 14.5 in the $\mathrm{T} 1 \mathrm{w}$ image to 35.2 in the CSF-SP image $(+143 \%)$. Finally, contrast between WM and GM increased from a maximum of 7.5 in the T2w image to 11.5 in the CSF-SP image $(+53 \%)$. The additional contrast in CSF-SP images may aid the quantification and analysis of lesion activity in MR exams of MS patients.
\end{abstract}

\section{Introduction}

MRI provides very sensitive detection of the lesions of Multiple Sclerosis (MS)[1]. We are developing new techniques to provide information about temporal changes in MS lesion intensity composition from sequential multispectral MR exams of MS patients[2]. Our methods are based upon analysis of the multispectral "feature space" distributions of tissue and lesion intensities[3]. However, as the number of images in each exam increases the feature space dimension also increases making feature space visualization and analysis more difficult.

In this paper we present a new method to extract data from multidimensional feature spaces while minimizing the loss of information. Our new technique provides images of "spectral phase" (SP) relative to a reference tissue. SP images have a number of important advantages: a) they are largely insensitive to intensity inhomogeneities in the multispectral MR exam; b) they allow retrospective suppression of reference tissue signals; c) they provide information about the underlying MR characteristics of lesions and other tissues while retaining high spatial resolution; and, d) they can provide tissue contrast much greater than any of the MR images. In this paper we describe the construction of spectral phase images relative to cerebro-spinal fluid (CSF) and show their application to MR exams of five MS patients selected from a clinical trial underway at our institute.

C. Taylor, A. Colchester (Eds.): MICCAI'99, LNCS 1679, pp. 218-226, 1999.

(C) Springer-Verlag Heidelberg Berlin 1999 


\section{Methods}

Multispectral analysis was performed on MR exams of five MS patients selected at random from a clinical trial underway at our institute. Patients were imaged on a 1.5T Signa scanner (General Electric Systems). Four MR contrasts at each of 24 slices covering the head were acquired from each patient using spin echo imaging: proton density weighted (PDw); T2 weighted (T2w); T1 weighted (T1w); and, T1 weighted after administration of gadolinium-DTPA contrast agent (GAD). Imaging parameters were as follows: $\mathrm{FOV}=22 \times 16.5 \mathrm{~cm}$.; slice thickness $=5 \mathrm{~mm}$. with no gap; matrix $=256 \mathrm{x}$ 192; flow compensation was enabled. For the proton density/T2 weighted images, TR/ TE1/TE2 = 5000/30/80 msec; BW1/BW2 = 15.6/7.81 khz; 1 nex. Total scan time was 10 minutes 24 seconds. For the T1 weighted images TR $=550 \mathrm{msec}$; TE $=13 \mathrm{msec}$; $\mathrm{BW}=16 \mathrm{khz} ; 2$ nex. Total scan time was 5 minutes 23 seconds. After the initial T1 acquisition $0.1 \mathrm{mmol}$ per $\mathrm{kg}$ gadolinium-DTPA was injected intravenously, without disturbing the patient setup. After 5 minutes a second gadolinium enhanced T1 weighted sequence was acquired with the same parameters as above. A standard patient setup and exam slice orientation procedure was used to minimize patient motion between the PD/T2w and T1w scans and provide slice positioning which varied less than $1 \mathrm{~mm}$ over the entire patient volume.

Images were transferred to a SUN Ultrasparc 10 workstation (Sun Microsystems, Mountainview, California) for preprocessing. Initially, intensity non-uniformity in each multispectral exam was corrected using the non-parametric, non-uniform intensity normalization algorithm developed by Sled et. al[4]. Next, a non-linear anisotropic diffusion based filter was applied to improve the signal-to-noise ratio within the images. The filter is based on one reported by Perona and Malik[5] but extended to incorporate information from an arbitrary number of spectral bands when calculating the diffusion coefficient. This filter requires two input parameters: the number of iterations; and, an estimate of image noise. We selected three iterations as suggested by Gerig et al[6]. Image noise was estimated using twice the standard deviation measured in a large region of interest (ROI) placed in air. Although signal in air typically follows a Rician distribution, it can be used to predict the Gaussian distribution of signals in tissues[7]. Finally, in each exam four slices covering the lateral ventricles were selected for processing. A single 4-D feature space was constructed for each patient from the four MR exam intensities in the selected slices.

The 4-D feature spaces were used to calculate spectral phase images relative to cerebro-spinal fluid (CSF). CSF was selected as a reference signal since it is easily identifiable, allows definition of large homogenous ROI's and has stable MR image signal through disease progression. ROI's were placed were placed within the lateral ventricles of each patient. ROI's were placed well away from the ventricle edges to reduce any partial volume effects between slices. For each patient 'p' a CSF reference intensity vector was formed as follows: 


$$
\overrightarrow{\mathrm{CSF}}_{p}=\left[\begin{array}{llll}
\overline{\mathrm{R}}_{\mathrm{PDw}, p} & \overline{\mathrm{R}}_{\mathrm{T} 2 \mathrm{w}, \mathrm{p}} & \overline{\mathrm{R}}_{\mathrm{T} 1 \mathrm{w}, \mathrm{p}} & \overline{\mathrm{R}}_{\mathrm{GAD}, \mathrm{p}}
\end{array}\right]^{\top}
$$

where

$$
\overline{\mathrm{R}}_{\mathrm{b}, \mathrm{p}}=\frac{\sum_{\mathrm{ij}} \mathrm{I}_{\mathrm{bijp}}}{\mathrm{n}_{\mathrm{p}}}, \mathrm{ij} \in \mathrm{ROI}, \mathrm{b} \in\{\mathrm{PDw}, \mathrm{T} 2 \mathrm{w}, \mathrm{T} 1 \mathrm{w}, \mathrm{GAD}\}
$$

given that: $I_{b i j p}$ is the voxel value from spectral band ' $b$ ' in voxel 'ij' within patient ' $p$ '; and, $n_{p}$ is the number of voxels in the CSF ROI. Here ' $i, j$ ' were restricted to indicate voxels within the CSF ROI.

Spectral phase images for each patient were then constructed by determining the CSF-relative spectral phase at every exam location ' $x y$ ':

$$
\mathrm{CSF}-\mathrm{SP}_{\mathrm{xyp}}=\overrightarrow{\mathrm{CSF}}_{\mathrm{p}} \bullet \vec{\nabla}_{\mathrm{xyp}}
$$

where

$$
\nabla_{x y p}=\left[I_{b x y p}\right]^{\top}, b \in\{P D w, T 2 w, T 1 w, G A D\}
$$

given that: $I_{b x y p}$ is the voxel value from spectral band ' $b$ ' in voxel 'xy' within patient 'p'. With this algorithm tissues which are spectrally "close" to CSF have small values in the output image, while those that are "far" from CSF have high values.

Contrast in the original MR and CSF-SP images was determined from the signal difference to noise ratio (dSNR) between: CSF and normal-appearing white matter (NAWM); CSF and gray-matter (GM); and NAWM and GM. dSNR between pairs of tissues was calculated using the following equation:

$$
\mathrm{dSNR}=\frac{\left|\overline{\mathrm{x}}_{1}-\overline{\mathrm{x}}_{2}\right|}{\sqrt{\frac{\left(\mathrm{n}_{1}-1\right) \cdot \mathrm{s}_{1}^{2}+\left(\mathrm{n}_{2}-1\right) \cdot \mathrm{s}_{2}^{2}}{\mathrm{n}_{1}+\mathrm{n}_{2}-2}}}
$$

where: $\bar{x}_{i}$ is the mean signal measured within each tissue ROI; $s_{i}^{2}$ is the variance within each tissue ROI; and $n_{i}$ is the number of voxels within each ROI. Note that this is similar to a two-sample unpaired t-test under the assumption of equal variances[8]. The mean and standard deviation of the dSNR measurements across the 5 patients were then plotted and compared. 


\section{Results}

Figure 1 shows PDw, T2w, T1w and contrast enhanced T1w images from a single slice in Patient 1 . The lesion burden in this patient is very small with only one prominent lesion on the anterior horn of the left lateral ventricle. This lesion is not readily visible in the T1 weighted image nor in the gadolinium enhanced image. These images are typical of those for patients in the early stages of disease.
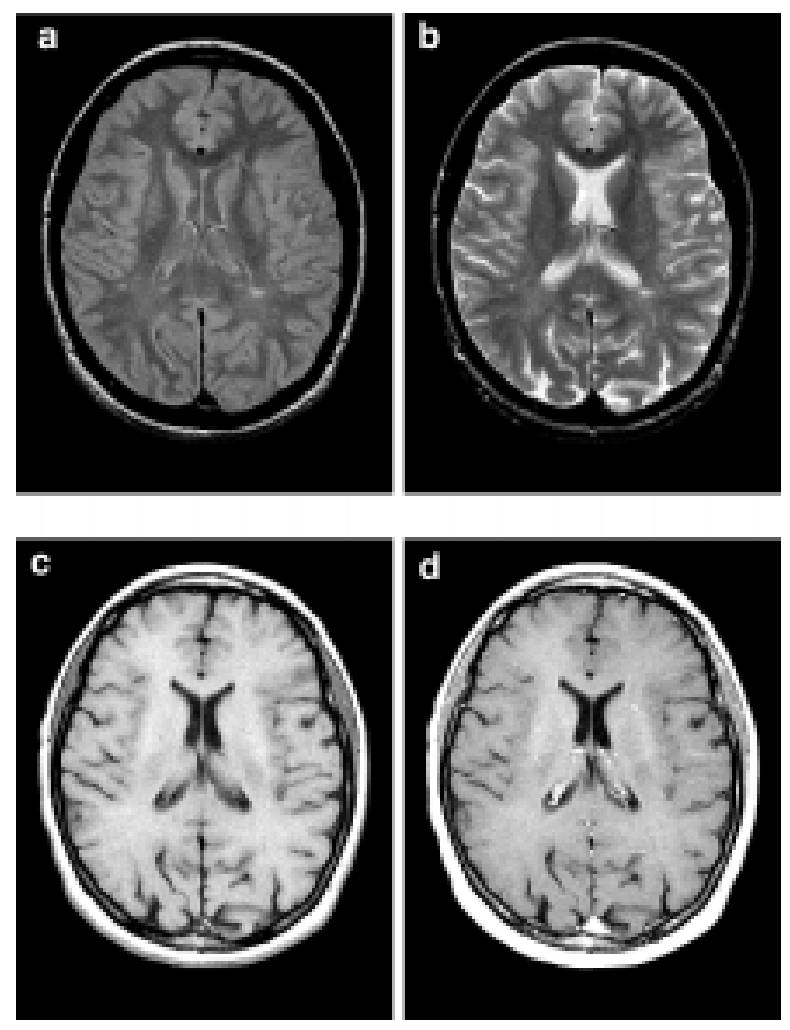

FIG. 1. a) through $d$ ) are respectively spin-echo PDw, T2w, T1w, and T1w after administration of gadolinium-DTPA contrast agent. This patient has one lesion near the anterior horn of the left lateral ventricle. However, this lesion does not enhance after administration of contrast agent. These images are typical of those analyzed in these experiments

Figure 2 shows the CSF-SP image calculated at the same slice as the images in Figure 1. The image has high contrast, with an overall appearance similar to anatomical images. Indeed, some white-matter tracks are visible. Figure 3 shows tissue contrasts measured between CSF and NAWM; CSF and GM; and, NAWM and GM, in the MR and CSF-SP images from the $5 \mathrm{MS}$ patient exams. The CSF-SP images provided higher tissue contrast than any of the MR images. Part of the increased contrast resulted from application of the anisotropic diffusion filter algorithm which reduced the standard deviation within tissues by $33 \%$ on average. In turn, this increased dSNR 
between tissues by $33 \%$ on average. However, calculation of CSF-SP images increased tissue contrast further: CSF-NAWM contrast was increased from a maximum of 19.5 in the T1w image to 56 in the CSF-SP image (+187\%). CSF-GM contrast increased from a maximum of 14.5 in the T1w image to 35.2 in the CSF-SP image (+143\%). Finally, NAWM-GM contrast increased from a maximum of 7.5 in the T2w image to 11.5 in the CSF-SP image $(+53 \%)$.

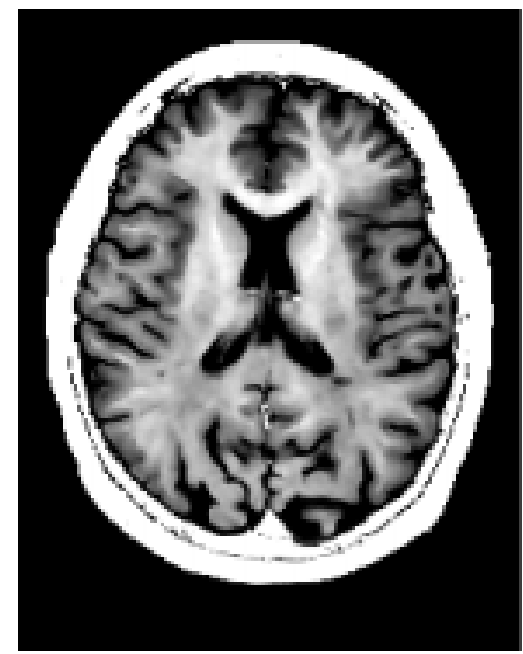

FIG. 2. Spectral phase images not only provide high contrast, but also allow selective suppression of tissue signals during post-processing.This figure shows a cross-sectional spectral phase image relative to cerebro-spinal fluid (CSF-SP). Using this technique to suppress fluid signal produces images with tissue contrasts similar to anatomical images

The increased contrast in CSF-SP images may allow better visualization of the heterogeneity within some lesions. Figure 4 shows close-up views of a single periventricular lesion in Patient 2.The CSF-SP image reveals heterogeneity within the lesion region which is not apparent in either the PDw or T2w images. Much of the lesion is not visible in the $\mathrm{T} 1 \mathrm{w}$ images. Visualization of subtle changes in contrast within and around the lesion region in CSF-SP images may provide a sensitive indication of change, and thus disease activity.

\section{Discussion}

A number of authors have shown that analysis of multi-dimensional feature spaces can aid the identification and detection of various pathologies. For example Vinitski et al have shown that cluster classification in a 3-dimension feature space improves tissue segmentation compared to classification in a 2-dimensional feature space[9]. They have also shown that utilization of a 3-dimensional feature space allows identification of additional tissues within tumor regions which correlate with histologic samples[10]. 


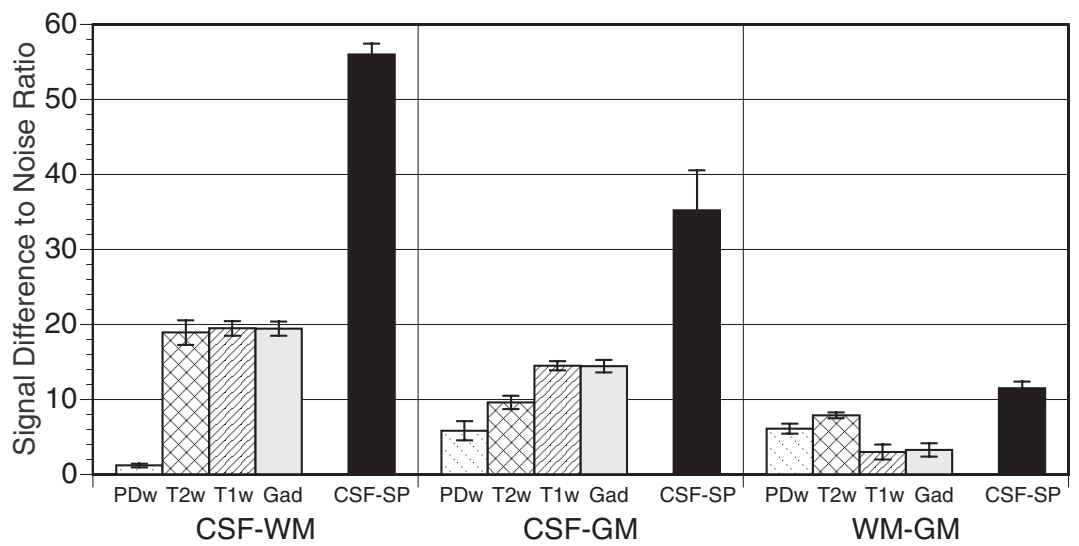

FIG. 3. The mean signal-difference to noise ratios (dSNR) measured between tissues in MR and CSF-SP images from 5 MS patients. The error bars indicate one standard error about each mean. CSF spectral phase images provide better dSNR between CSF, normal-appearing white matter (WM) and gray matter (GM) than any of the MR images. The mean dSNR between CSF and WM in CSF-SP images is 56, while it is only 20 at most the MR images. The improved contrast may aid detection of subtle changes in lesions over time
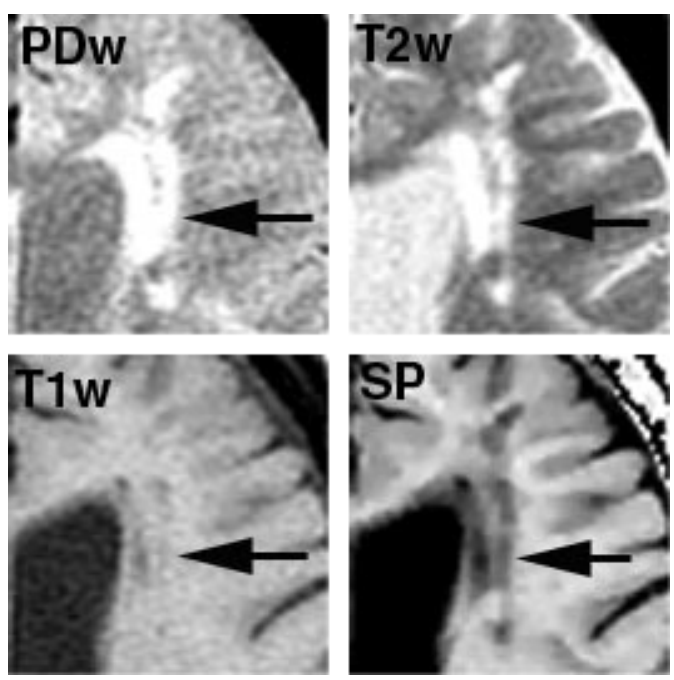

FIG. 4. Increased contrast in CSF spectral phase images may aid the visualization of lesion heterogeneity. The images are close-up views of a large lesion in the original proton-density weighted $(P D w)$, T2 weighted $(T 2 w)$, T1 weighted $(T 1 w)$ and spectral phase relative to cerebrospinal fluid $(S P)$ images. The lesion (arrow) is barely visible in the T1w image, and appears relatively homogenous in the PDw and T2w images. The CSF-SP image reveals structure within the lesion region. Notice also the good suppression of CSF signal in the SP image 
They speculate that analysis of additional contrast (i.e. analysis of higher dimensional feature spaces) may improve segmentation further.

However visualization and analysis of feature spaces becomes more difficult as their dimension increases. One solution is to use tissue class information to construct a transformation from a higher dimensional feature space down to a 2-D or 3-D feature space. Zadeh et. al. have shown one such transformation which has the added benefit of increasing class separability[11],[12].

Brunetti et. al. describe a different technique which combines multi-spectral MRI data into a quantitative color image[13]. They found that this new color image helped improve the detectability of white matter lesions in multiple sclerosis. It also improved the agreement and reduced the variability between radiologists compared to conventional spin echo studies. However their technique incorporates information from exactly three MR images and is therefore incapable of incorporating information from additional contrast.

In this paper we have presented a new technique to extract information from multidimensional feature spaces constructed from MR images of patients with multiple sclerosis. Our new technique provides images of spectral phase relative to CSF. CSF was selected as the reference signal since it is easily identifiable and maintains intensity characteristics through disease progression. However, alternative reference signals could be selected from other tissues, or phantom materials within the exam volume and may provide even better discrimination of lesions and normal tissues. A more extensive study needs to be performed to determine the merit of alternative reference signals.

CSF-SP images can combine information from an arbitrary number of MR image contrasts. This may be useful for simplifying or reducing a complex data set down to a single image. CSF-SP images also allow retrospective suppression of reference tissue signals. This may be useful if the reference tissue obscures or has contrast similar to another tissue of interest in one or more of the MR images. Finally, CSF-SP images can provide tissue contrast much greater than that present in any of the MR images. This increased contrast may aid the visualization of lesion heterogeneity and improve the detection of subtle changes in lesions over time.

Figure 5 shows CSF-SP images calculated from MR exams acquired every 4 weeks over a 5 month period from an additional MS patient. Each image was produced within the same $3 \mathrm{~mm}$ thick slice. Although registration between exams is not perfect, this slice was selected for analysis since all lesions visible in this slice were also visible in the slices immediately superior and inferior to this slice. A number of diffuse abnormalities are visible in this image sequence which are not apparent in the original MR images. For example, a dark, diffuse region appears around a prominent lesion in the left anterior region on Day 26. Monitoring of subtle changes in CSF-SP images may provide a more sensitive indication of disease activity than analysis of standard MR exams of MS patients. However, an ROC analysis comparing the detectability of MS 
lesions in conventional and CSF-SP images must be performed to demonstrate this point conclusively.
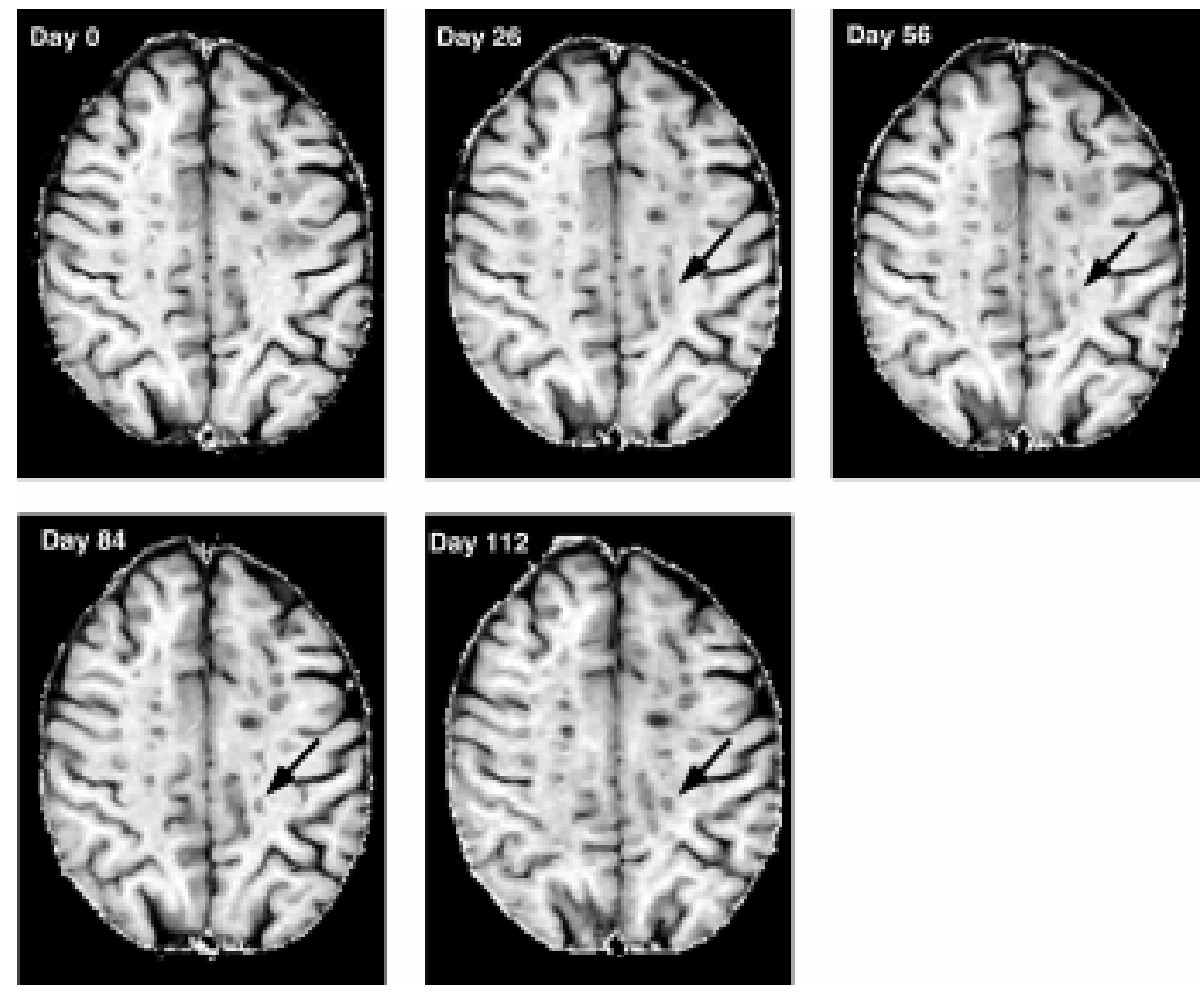

FIG. 5. Spectral phase images may indicate subtle changes in MR exam intensities over time. These are CSF-SP images derived from MR exams acquired over 5 months within the same $3 \mathrm{~mm}$ thick axial cross-section from an additional MS patient. Note, for example, the growth of a diffuse abnormality in the lower right quadrant by Day 26 (arrow). This abnormality recedes by Day 84 leaving two small focal lesions

\section{Conclusions}

A new technique has been developed to calculate "spectral phase" images from multispectral MR exams of MS patients. The technique provides a convenient way of reducing multispectral MR exams to a single image. Spectral phase images relative to CSF provide better tissue contrast than that found in any of the MR images. The increased contrast may aid the quantification and analysis of lesion activity in MR exams of MS patients. 


\section{Acknowledgments and References}

This work was supported by the Canadian Multiple Sclerosis Society.

1 Evans AC, Frank JA, Antel J, Miller DH "The role of MRI in clinical trials of multiple sclerosis: comparison of image processing techniques”. Ann. Neurol., 41(1), 125-132 (1997).

2 M.W. Vannier et. al, "Multispectral analysis of magnetic resonance images". Radiology, 154:221-224, (1985).

3 Mitchell JR, Jones C, Karlik SJ, Kennedy K, Lee DH, Rutt B and Fenster A. "MR multispectral analysis of multiple sclerosis lesions." Journal of Magnetic Resonance Imaging; 7:499-511 (1997).

4 Sled JG, Zijdenbos AP and Evans AC. "A nonparticipant method for automatic correction of intensity nonuniformity in MRI data". IEEE Transactions on Medical Imaging; 17(1):87-97 (1998).

5 Perona P, Malik J. "Scale-space and edge detection using anisotropic diffusion." IEEE Transactions on Pattern Analysis and Machine Intelligence 12(7):629-639 (1990)

6 Gerig G., Kubler O, Kikinis R, Jolesz FA, "Nonlinear anisotropic filtering of MRI data." IEEE Transactions on Medical Imaging, 11 (2), 221-232, (1992).

7 Gudbjartsson H, Patz S. "The Rician distribution of noisy MRI data” Magnetic Resonance in Medicine; 34(6):910-914 (1995).

8 Armitage P, Berry G. Statistical methods in medical research. 2nd edition. Oxford: Blackwell Scientific Publications. (1990)

9 Vinitski S, Gonzalez C, Andrews D, Knobler R, Curtis M, Mohamed F, Gordon J and Khalili K. "In vivo validation of tissue segmentation based on a 3D feature map using both a hamster brain tumor model and stereotactically guided biopsy of brain tumors in man". Journal of Magnetic Resonance Imaging; 8(4):814-819 (1998).

10 Viniski S, Gonzalez, Mohamed P, Iwanga T, Knobler R, Khalili K and Mack J. "Improved intracranial lesion characterization by tissue segmentation based on a 3D feature map". Magnetic Resonance in Medicine; 37(3):457-469 (1997).

11 Soltanian-Zadeh H, IEEE Member, Windham JP and Peck DJ. "Optimal linear transformation for MRI feature extraction”. IEEE Transactions on Medical Imaging; 15(6):749-767 (1996).

12 Soltanian-Zadeh H, Peck DJ, Windham JP and Mikkelsen T. "Brain tumor segmentation and characterization by pattern analysis of multispectral NMR images". Nuclear Magnetic Resonance in Biomedicine; 11:201-208 (1998).

13 Brunetti A, Tedeschi G, Di Costanzo A, Covelli EM, Aloj L, Bonavita S, Ciarmiello A, Alfano B and Salvatore M. "White matter lesion detection in multiple sclerosis: improved interobserver concordance with multispectral MRI display.” Journal of Neurology; 244:586590 (1997). 